\title{
Bilateral Endophthalmitis in a Patient with Bacterial Meningitis Caused by Streptococcus pneumoniae
}

\author{
Hidemasa Torii ${ }^{a, c}$ Hiroshi Miyatac Eiko Sugisaka ${ }^{a, c}$ Yuho Ichikawa ${ }^{\text {a, c }}$ \\ Kei Shinoda ${ }^{a}$ b Makoto Inoue $^{a}$ \\ aDepartment of Ophthalmology, Keio University School of Medicine, and ${ }^{b}$ Laboratory of Visual Physiology, \\ National Institute of Sensory Organs, National Organization Tokyo Medical Center, Tokyo, and ${ }^{\mathrm{C}}$ Department of \\ Ophthalmology, Yokohama Municipal Citizen's Hospital, Yokohama, Japan
}

\author{
Key Words \\ Endophthalmitis - Meningitis • Proliferative \\ vitreoretinopathy $\cdot$ Streptococcus pneumoniae • \\ Vitreoretinal surgery
}

ommend that endogenous endophthalmitis be suspected in cases of meningitis, and if present, intravitreal and subconjunctival antibiotics should be promptly given to preserve vision.

Copyright $\odot 2008$ S. Karger AG, Basel

\begin{abstract}
Aim: To present a patient who developed bilateral endogenous endophthalmitis secondary to Streptococcus pneumoniae meningitis. Methods: A 44-year-old man who was unconscious because of bacterial meningitis was referred to us for conjunctival hyperemia and decreased pupillary light reflexes. Ophthalmoscopy revealed inflammation in the anterior chamber and vitreous opacities in both eyes. He was diagnosed as having endogenous endophthalmitis associated with the meningitis. Ceftazidime and vancomycin hydrochloride were injected intravitreally and subconjunctivally repeatedly in both eyes. Results: Vision improved to 20/200 in the left eye 1 month later, but the right eye became hypotonic and blind. Vision deteriorated to light perception in the left eye due to a retinal detachment with proliferative vitreoretinopathy, and vitreous surgery successfully reattached the retina. Vision recovered to $20 / 80$, and the retina has remained attached for 1 year. Conclusions: We rec-
\end{abstract}

(c) 2008 S. Karger AG, Basel $0030-3755 / 08 / 2225-0357 \$ 24.50 / 0$

Fax +41613061234 E-Mail karger@karger.ch www.karger.com www.karger.com/oph

\section{Introduction}

Bacterial meningitis is a deadly disease due to central nervous system complications and is mainly caused by Streptococcus pneumoniae [1]. The ocular prognosis of endogenous endophthalmitis caused by $S$. pneumoniae is poor due to the inflammatory responses evoked by streptococcal exotoxins and enzymes [2-4]. A comprehensive search of the literature did not extract any reports of cases of endogenous endophthalmitis caused by $S$. pneumoniae that retained useful vision after treatment. We describe a patient with bilateral endogenous endophthalmitis associated with meningitis who developed hypotony and blindness in 1 eye but the vision was successfully rescued by antibiotics and vitreous surgeries for a subsequent retinal detachment and severe hypotony in the other eye. 

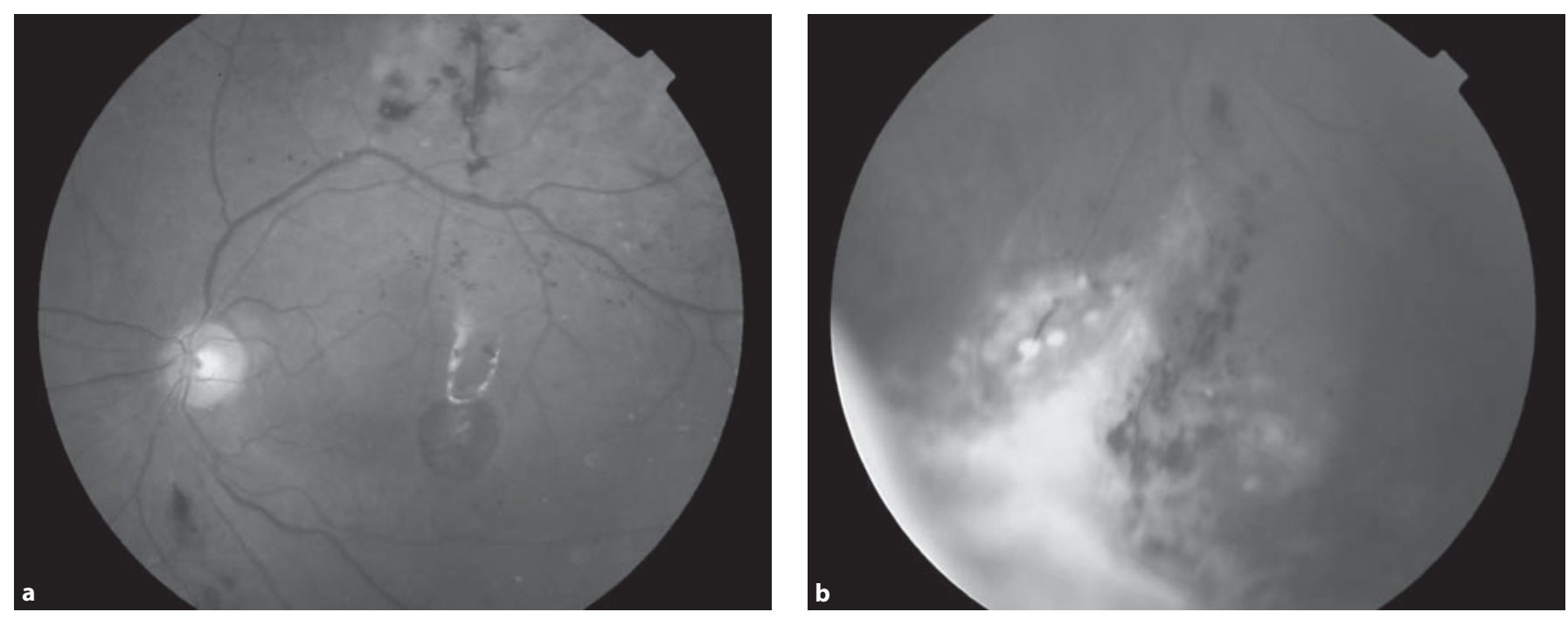

Fig. 1. Fundus photographs of the left eye after the surgeries. a The retina is reattached but with a macular hole under silicone oil tamponade. Intraocular pressure was $10 \mathrm{~mm} \mathrm{Hg}$. b Infectious foci are seen inferiorly as whitish fibrous membrane surrounded by atrophic retinal breaks treated with endolaser photocoagulation.

\section{Case Report}

A 44-year-old man developed a fever on May 14, 2005. Although intravenous fosfomycin and antipyretic drugs were given, he suddenly fell into a coma 5 days later. His white blood cell count was $21,200 / \mu \mathrm{l}$, and C-reactive protein was $11.8 \mathrm{mg} / \mathrm{dl}$. Glucose was not detected in the cerebrospinal fluid, but the mononuclear and polynuclear cell counts were $1,653 / \mu \mathrm{l}$ and $10,872 / \mu \mathrm{l}$, respectively. He was diagnosed as having bacterial meningitis and was treated with systemic ampicillin (12 g/day) and cefotaxime $(8 \mathrm{~g} /$ day). These antibiotics were supplemented with glycerol $(400 \mathrm{ml} /$ day) and dexamethasone (6 mg/day) to avoid cerebral edema.

S. pneumoniae was detected in cultures of both blood and cerebrospinal fluid on May 23, 2005. The blood and cerebrospinal fluid samples were cultured on both anaerobic and aerobic culture plates and also on fungus culture media. The fungus cultures were negative. Ophthalmological examination was performed on the same day because of conjunctival hyperemia and decreased pupillary reflexes, but his visual acuity could not be measured because he was in a coma. Ophthalmoscopy revealed bilateral inflammation in the anterior chamber and opacities in the vitreous, but the intraocular pressure was already low in the right eye by tactile compression. He was diagnosed as having endogenous endophthalmitis associated with the meningitis and anterior segment necrosis in the right eye. Intravitreal injections of ceftazidime $(2.25 \mathrm{mg})$ and vancomycin hydrochloride $(1 \mathrm{mg})$ combined with subconjunctival injections of ceftazidime $(100 \mathrm{mg})$ and vancomycin hydrochloride $(25 \mathrm{mg})$ were given every other day.

The inflammation in the anterior chamber and vitreous opacity in the left eye decreased 2 weeks later, but vision remained at light perception in each eye. The intraocular pressure was $0 \mathrm{~mm}$ $\mathrm{Hg}$ in the right eye and $2 \mathrm{~mm} \mathrm{Hg}$ in the left eye, and the right eye developed phthisis bulbi. Vision improved to 20/200 in the left eye 1 month later with a decrease in the vitreous opacities, but infectious foci were observed in the inferior retina.
On June 27, 2005, the vision in his left eye deteriorated to light perception, and ophthalmoscopy revealed a total retinal detachment with proliferative vitreoretinopathy. Vitreous surgery was performed under general anesthesia on July 1. After lensectomy, the vitreous opacities were removed, and an epiretinal membrane was peeled off the retina around the infectious foci with atrophic retinal breaks. These atrophic breaks were treated with endophotocoagulation, and an encircling buckling with a silicone tire was exoplanted. Silicone oil was injected into the vitreous at the end of the procedure because the patient had difficulties in maintaining a face-down position.

The retina in the left eye was reattached, and the vision improved to hand movements. A macular hole developed 1 week later, but the patient did not report any visual deterioration. However, the retina redetached, and vision deteriorated to light perception. Another surgery was performed with membrane peeling and reinjection of silicone oil. The retina was successfully reattached under the silicone oil, and vision has remained 20/80 in the left eye for 1 year with normal intraocular pressure (fig. 1).

\section{Discussion}

The Endophthalmitis Vitrectomy Study group examined the efficacy of immediate vitrectomy and intravitreal antibiotics in the management of postoperative bacterial endophthalmitis after cataract surgery. They reported that there was no benefit in the visual outcome whether a vitrectomy was performed immediately or not except in cases of endophthalmitis with light perception vision which should have immediate pars plana vitrectomy [5]. Furthermore, the Endophthalmitis Vitrectomy Study 
group reported no overall ocular benefit from the administration of systemic antibiotics. However, cases with definitive endogenous endophthalmitis were not studied by the Endophthalmitis Vitrectomy Study group.

Endogenous endopthtalmitis can develop from the spread of infections from other sites [3]. The importance and efficacy of a mixture of systemic antibiotics have been documented in patients with metastatic endophthalmitis $[3,6]$. However, the poor prognosis of endogenous endophthalmitis caused by $S$. pneumoniae has also been reported in spite of the relatively good sensitivity of $S$. pneumoniae to antibiotics. Miller et al. [2] reported on 25 eyes with endophthalmitis caused by $S$. pneumoniae and on 2 eyes with endogenous endophthalmitis, and both of these 2 eyes ended up with no light perception. They reviewed the endogenous endophthalmitis caused by $S$. pneumoniae reported in the literature, and they found that one half of the cases were immunocompromised or asplenic or both. Others have also described cases of endophthalmitis caused by $S$. pneumoniae [7, 8]. However, all of these eyes also progressed to no light perception. The poor prognosis of endophthalmitis is probably due to the streptococcal exotoxins and enzymes which rapidly destroy the cellular membranes of the iris and ciliary epithelial cells [1]. This then leads to cellular destruction, hypotony and anterior segment necrosis [4]. In addition, severe systemic conditions, such as coma due to meningitis, deter early detection and treatment. There was a possibility of a contamination of the bacterial culture and a possible Candida infection even though $S$. pneumoniae was positive in both blood and cerebrospinal fluid with low glucose levels and intense infiltration of inflammatory cells in the cerebrospinal fluid and negative fungus cultures. However, a very high incidence of pathogen in septicemia caused by $S$. pneumoniae has been described if the blood culture was positive for it [9].

We recommend that moribund patients with systemic infections have their eyes examined, because they are not able to complain about visual symptoms. The failure to examine the eyes of septic patients who are not able to communicate can allow endogenous endophthalmitis to go undetected and progress to blindness. In fact, the right eye in our case also rapidly developed phthisis after the initial ocular examination. S. pneumoniae might have spread to both eyes despite intensive antibiotic treatment because of the retarded course of the endophthalmitis in the current case. Thus, immediate treatments by intravitreal and subconjunctival injections of a mixture of systemic antibiotics are recommended after the diagnosis of metastatic endophthalmitis. However, Smiddy et al. [10] reported that subconjunctival antibiotics might not be necessary to treat postoperative endophthalmitis after cataract surgery, and only intravitreal and topical antibiotics are necessary. In addition, the efficacy of intravitreal antibiotics has not been proved for endogenous endophthalmitis in which most cases were caused by Klebsiella pneumoniae [11]. While ours is only 1 case, the early recognition and immediate treatments by intravitreal and subconjunctival injections of antibiotics combined with vitreous surgery for ancillary complications are essential to preserve vision in cases of endogenous endophthalmitis with bacterial meningitis.

\section{References}

1 Koedel U, Scheld WM, Pfister HW: Pathogenesis and pathophysiology of pneumococcal meningitis. Lancet Infect Dis 2002;2: 721-736.

2 Miller JJ, Scott IU, Flynn HW Jr, Smiddy WE, Corey RP, Miller D: Endophthalmitis caused by Streptococcus pneumoniae. Am J Ophthalmol 2004;138:231-236.

-3 Greenwald MJ, Wohl LG, Sell CH: Metastatic bacterial endophthalmitis: a contemporary reappraisal. Surv Ophthalmol 1986;31: 81-101.

4 Garretson BR, Aragones JV: Anterior segment necrosis associated with Streptococcus pneumoniae meningitis. Retina 1999;19: 163-164.
5 Endophthalmitis Vitrectomy Study Group: Results of the Endophthalmitis Vitrectomy Study: a randomized trial of immediate vitrectomy and of intravenous antibiotics for the treatment of postoperative bacterial endophthalmitis. Arch Ophthalmol 1995;113: 1479-1496.

6 Chan SM, Hodge WG, Leonard BC: Postoperative Streptococcus pneumoniae endophthalmitis complicated by meningitis. Arch Ophthalmol 1998;116:951-953.

7 De Groot V, Stempels N, Tassignon MJ: Endogenous pneumococcal endophthalmitis after splenectomy: report of two cases. Bull Soc Belge Ophtalmol 1992;243:147-151.

8 Piczenik Y, Kjer B, Fledelius HC: Metastatic bacterial endophthalmitis: a report of four cases all leading to blindness. Acta Ophthalmol Scand 1997;75:466-469.
-9 Weinstein MP, Towns ML, Quartey SM, Mirrett S, Reimer LG, Parmigiani G, Reller LB: The clinical significance of positive blood cultures in the 1990s: a prospective comprehensive evaluation of the microbiology, epidemiology, and outcome of bacteremia and fungemia in adults. Clin Infect Dis 1997;24:584-602.

10 Smiddy WE, Smiddy RJ, Ba'Arath B, Flynn HW Jr, Murray TG, Feuer WJ, Miller D: Subconjunctival antibiotics in the treatment of endophthalmitis managed without vitrectomy. Retina 2005;25:751-758.

11 Wong JS, Chan TK, Lee HM, Chee SP: Endogenous bacterial endophthalmitis: an east Asian experience and a reappraisal of a severe ocular affliction. Ophthalmology 2000; 107:1483-1491. 